\title{
Muscle Oxygen Extraction Is Higher during Constant Work Exercise than during Incremental Ramp Exercise
}

\author{
Junshiro Yamamoto ${ }^{1}$ and Kazuyuki Tabira ${ }^{1}$ \\ ${ }^{1}$ Division of Health Science, Graduate School of Health Science, Kio University, Kitakatsuragi-gun, Nara, Japan
}

\begin{abstract}
A cardiopulmonary exercise test (CPX) can provide objective measures of exercise capacity. Specifically, incremental ramp exercise (IRE) and constant work-rate exercise (CWE) protocols are frequently used in clinical practice and for research. The CWE endurance time has shown larger increases than other indexes assessed by IRE after rehabilitation intervention. Muscle oxygen extraction is one of the important physiological factors of exercise capacity; however, the differences in muscle oxygen kinetics between IRE and CWE remain unclear. The purpose of this study was to compare the muscle oxygen kinetics during IRE and CWE. Each of the 15 participants performed IRE and CWE to exhaustion on a cycle ergometer. Ventilatory and muscle deoxygenation responses were measured during the tests; muscle deoxygenation was determined using near-infrared spectroscopy. No differences in oxygen uptake and heart rate were observed between the two tests. A comparison of the muscle deoxygenation kinetics between the two tests indicated significantly greater deoxygenation during the CWE than during the IRE at all time points $(p<$ 0.05). The muscle deoxygenation kinetics, as percentages of maximal oxygen uptake $\left(\dot{\mathrm{V}} \mathrm{O}_{2 \max }\right)$, were higher during CWE than during IRE, except at $80 \%$ of $\dot{V}_{2 \max }(p<0.05)$. These results suggest that skeletal muscle during CWE extracts oxygen at a rate comparable to that during IRE, and that exercise capacity assessed using CWE might be linked to a higher overall $\mathrm{O}_{2}$ extraction. The fact that endurance time during CWE is more sensitive to rehabilitation intervention may be due to improvements in muscle oxygen extraction.
\end{abstract}

Keywords: cardiopulmonary exercise test; constant work-rate exercise; incremental ramp exercise; muscle deoxygenation; near-infrared spectroscopy

Tohoku J. Exp. Med., 2014 May, 233 (1), 57-63. (C) 2014 Tohoku University Medical Press

\section{Introduction}

Cardiopulmonary exercise testing (CPX) is the gold standard for evaluating the cause of exercise intolerance in patients with pulmonary and cardiac disease (Palange et al. 2007). CPX can provide an objective measure of exercise capacity, identify the mechanisms limiting exercise tolerance, establish indices of the patient's prognosis, and monitor disease progression and/or the response to interventions (Palange et al. 2007). Incremental ramp exercise (IRE), the most widely used CPX protocol in clinical practice (American Thoracic Society; American College of Chest Physicians 2003), is used to calculate aerobic threshold (AT), respiratory compensation point, peak work rate $\left(\mathrm{WR}_{\text {peak }}\right)$, and maximal oxygen uptake $\left(\dot{\mathrm{V}} \mathrm{O}_{2 \max }\right)$. On the other hand, the constant work-rate exercise (CWE) is useful for the analysis of gas exchange kinetics and dynamic hyperinflation. Endurance time using the CWE protocol has recently gained popularity (Casaburi 2005; Palange et al. 2007) because it shows larger increases than other indexes assessed by IRE, such as bronchodilation (Oga et al. 2000) and rehabilitation (Arizono et al. 2011), after specific interventions.

Although IRE and CWE can assess exercise tolerance and other limiting factors, the physiological responses at exhaustion might differ. During the IRE and CWE tests, $\dot{\mathrm{V}} \mathrm{O}_{2 \max }$ occurs at exhaustion (Sloniger et al. 1996; Hill et al. 2002). However, $\dot{\mathrm{V}} \mathrm{O}_{2 \max }$ is considered to be directly linked to the $\mathrm{O}_{2}$ delivery during IRE and to the rate of adenosine triphosphate (ATP) generation during CWE (Bassett and Howley 2000). Intramuscular phosphocreatine (PCr) levels attain a consistent nadir before or at the same time as exhaustion during CWE (Jones et al. 2008; Vanhatalo et al. 2010). IRE, however, attains a maximal work rate (WR) before the PCr nadir is reached (Hogan et al. 1999) in magnetic resonance spectroscopy studies. These observations suggest that the CWE test is restricted by muscle metabolism energy depletion. Thus, the physiological mechanisms limiting exercise tolerance are different for the 2 protocols, and an understanding of the physiological mechanisms

Received January 14, 2014; revised and accepted April 18, 2014. Published online May 15, 2014; doi: 10.1620/tjem.233.57.

Correspondence: Junshiro Yamamoto, Division of Health Science, Graduate School of Health Science, Kio University, 4-2-2 Umaminaka, Koryo-cho, Kitakatsuragi-gun, Nara 635-0832, Japan.

e-mail: j_yamamoto1046@yahoo.co.jp 
leading to exhaustion should enable more accurate assessments of exercise intolerance. However, the differences in muscle oxygen kinetics between IRE and CWE remain unclear.

Near-infrared spectroscopy (NIRS) provides a noninvasive measure of oxyhemoglobin $\left(\mathrm{O}_{2} \mathrm{Hb}\right)$ and deoxyhemoglobin $(\mathrm{HHb})$ levels in the microcirculation, and the derived NIRS signals reflect a balance between $\mathrm{O}_{2}$ delivery and utilization in the portion of the muscle under investigation; $\mathrm{HHb}$ values are considered similar to $\mathrm{O}_{2}$ extraction values (Grassi et al. 2003). Neary et al. (2001) reported that the degree of muscle deoxygenation during prolonged (23-32 min) CWE exceeded that observed during IRE. The report indicated that a longer duration of simulated exercise elicited greater muscle deoxygenation. It is necessary to investigate the differences in muscle deoxygenation during both tests for similar exercise durations to provide useful clinical interpretations. Therefore, this study compared muscle deoxygenation during CWE and IRE over similar exercise durations. We hypothesized that muscle deoxygenation at exhaustion during CWE would be greater than that during IRE because of progressive fatigue and a higher muscle metabolism energy consumption.

\section{Methods}

Study participants

Fifteen healthy men (anthropometric characteristics are presented in Table 1) participated in this study; none of the participants had routine fitness habits, and none were smokers. The participants were informed of the potential risks and discomforts associated with the experiments before giving their written consent to participate in the study; the study was approved by the ethical review committee of Kio University (Koryo-cho, Japan).

\section{Study design}

Over a 2-week period, the participants completed 5-6 laboratory visits, each approximately 2-h long, with at least 1 day of rest

Table 1. Subject characteristics and physiological parameters.

\begin{tabular}{|c|c|}
\hline Variables & \\
\hline Age (yr) & $23.2 \pm 3.4$ \\
\hline Hight (cm) & $169.7 \pm 5.2$ \\
\hline Weight (kg) & $62.6 \pm 4.7$ \\
\hline BMI & $21.7 \pm 1.3$ \\
\hline$\dot{\mathrm{V}} \mathrm{O}_{2 \text { max }}(\mathrm{L} / \mathrm{min})$ & $3.04 \pm 0.71$ \\
\hline$\dot{\mathrm{V}} \mathrm{O}_{2 \mathrm{AT}}(\mathrm{L} / \mathrm{min})$ & $1.59 \pm 0.31$ \\
\hline $\mathrm{WR}_{\text {peak }}(\mathrm{W})$ & $241 \pm 32$ \\
\hline $\mathrm{CP}(\mathrm{W})$ & $158 \pm 19$ \\
\hline $\mathrm{W}^{\prime}(\mathrm{kJ})$ & $12.7 \pm 5.6$ \\
\hline
\end{tabular}

Variables are presented as mean \pm S.D. $\dot{\mathrm{V}} \mathrm{O}_{2}$, oxygen uptake; AT, Anaerobic Threshold; $\mathrm{WR}_{\text {peak }}$, peak work rate; $\mathrm{CP}$, critical power; $\mathrm{W}^{\prime}$, the curvature constant of the hyperbola. between visits. Following familiarization with the equipment and procedures, the participants participated in the tests within a temperature-controlled $\left(23^{\circ} \mathrm{C} \pm 1{ }^{\circ} \mathrm{C}\right)$ laboratory. The participants were instructed to be well hydrated and rested, and to refrain from alcohol consumption $(24 \mathrm{~h})$ and food and caffeine ingestion $(3 \mathrm{~h})$ before each testing session.

\section{Exercise protocols}

All exercise tests were performed after $3 \mathrm{~min}$ of rest and $2 \mathrm{~min}$ of warm-up $(20 \mathrm{~W})$ by pedaling a cycle ergometer (Lode; Corival, Groningen, The Netherlands) at a pedal cadence of $80 \pm 5 \mathrm{rpm}$. The participants pedaled to exhaustion during each test; exercise intolerance was defined as the point at which a participant was unable to maintain a cadence $>70 \mathrm{rpm}$, and had a respiratory exchange ratio $(\mathrm{RER})>1.15$ and a heart rate $(\mathrm{HR})>90 \%$ of the age-predicted maximum (Poole et al. 2008).

IRE: Each participant performed the IRE (work rate [WR] $=25$ $\mathrm{W} / \mathrm{min}$ ) to the limit of tolerance in order to determine the $\dot{\mathrm{VO}}_{2 \max }$, $\mathrm{WR}_{\text {peak }}$, and other respiratory and metabolic variables.

Characterization of the power-limit of tolerance relationship: A randomized series of 3-4 CWE tests were performed to determine the power-limit of tolerance $\left(\mathrm{P}-\mathrm{T}_{\text {lim }}\right)$ relationship. Each test was performed at a different $\mathrm{P}$ output $\left(75 \%, 80 \%\right.$, and $\left.90 \% \mathrm{WR}_{\text {peak }}\right)$ to elicit intolerance within a period of approximately 2-15 min (Poole et al. 1988); therefore, when the $\mathrm{T}_{\lim }$ exceeded $15 \mathrm{~min}$, the participant worked for an additional trial $\left(100 \% \mathrm{WR}_{\text {peak }}\right)$.

Calculation of the $P-T_{\text {lim }}$ relationship: The limit of tolerance $\left(\mathrm{T}_{\text {lim }}\right)$ of severe-intensity exercise is well characterized as a hyperbolic function of the external power output $(\mathrm{P})$.

$$
\mathrm{W}^{\prime}=\mathrm{T}_{\lim } \times(\mathrm{P}-\mathrm{CP})
$$

where CP is the power asymptotes, termed "critical power", and $\mathrm{W}^{\prime}$ is the curvature constant of the hyperbola (Monod and Scherrer 1965; Moritani et al. 1981). CP and $\mathrm{W}^{\prime}$ were estimated from the intercept and slope, respectively, of the linear $\mathrm{P}_{-} \mathrm{T}_{\lim }$ relationship, using leastsquares linear regression (Poole et al. 1988):

$$
\mathrm{P}=\left(\mathrm{W}^{\prime} / \mathrm{T}_{\lim }\right)+\mathrm{CP} \text { (Fig. 1). }
$$

"Time-matched" CWE: The purpose of the present study was to compare muscle deoxygenation kinetics between IRE and CWE over a similar length of time. The time-matched CWE test was completed to exhaustion; based on the P-T $\mathrm{T}_{\lim }$ relationship, the WR was estimated to occur with the same time to exhaustion as during IRE.

\section{Measurements}

During the tests, we measured physiological responses, including ventilatory, metabolic, and muscle oxygenation variables. Ventilatory and metabolic variables were recorded breath-by-breath with a gas analyzer, and muscle oxygenation was recorded using NIRS.

Expiratory gas analysis: Ventilatory and metabolic variables were recorded breath-by-breath with a computerized metabolic cart (MetaMax3B; Cortex, Leipzig, Germany). $\dot{\mathrm{V}}_{2}$, minute ventilation $(\dot{\mathrm{V} E}), \mathrm{RER}$, and HR were determined from values averaged every $30 \mathrm{~s}$.

NIRS: Changes in the deoxygenation of the vastus lateralis muscle were evaluated by NIRS (BOM-L1TRW; Omegawave, Tokyo, Japan) at rest and during exercise. A NIRS probe was positioned on 


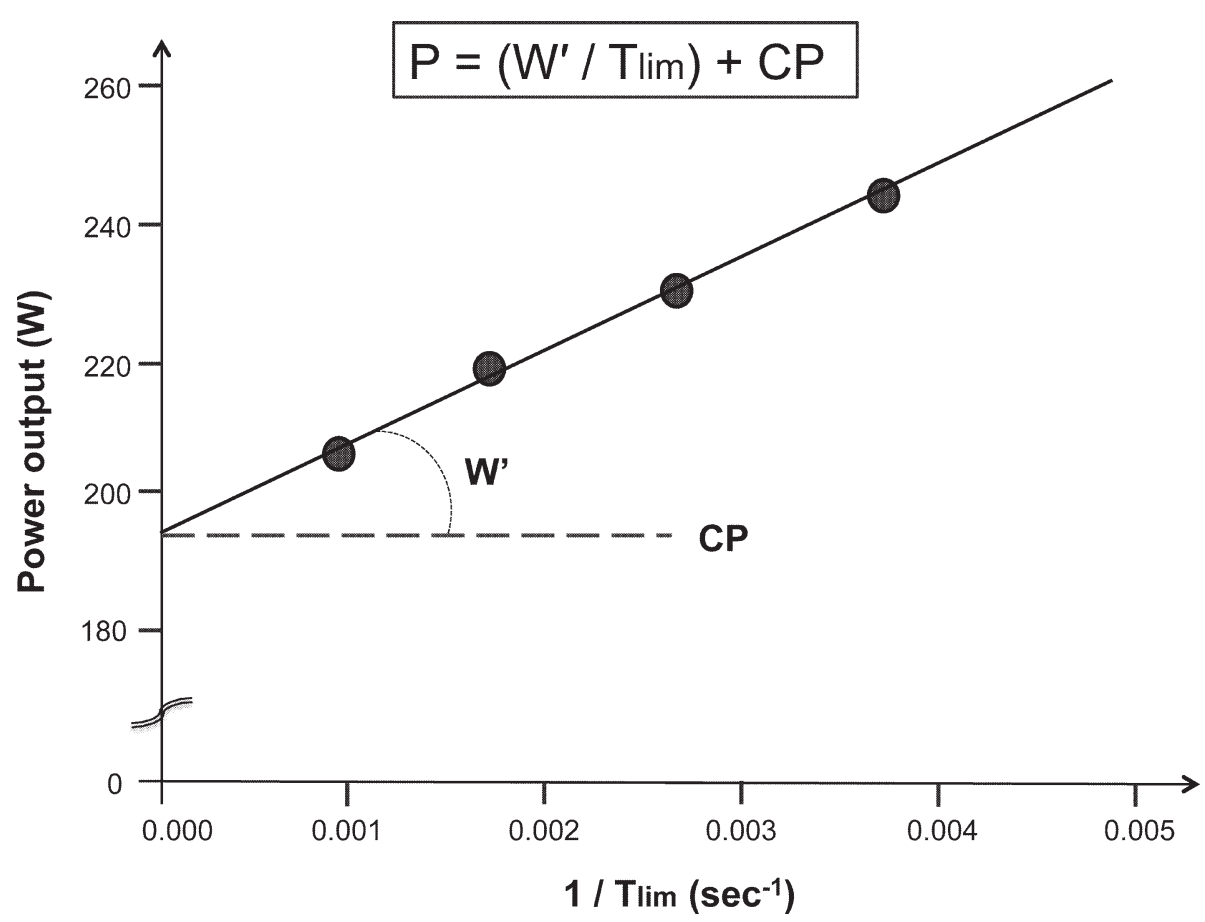

Fig. 1. Schematic of the power-limit of tolerance $\left(\mathrm{P}-\mathrm{T}_{\text {lim }}\right)$ relationship for severe-intensity exercise. The 4 dots indicate the time to exhaustion for independent tests at each power. The y-intercept represents the critical power $(\mathrm{CP})$ and the slope of the line represents the curvature constant of the hyperbola ( $\left.\mathrm{W}^{\prime}\right)$.

Table 2. Comparison of exercise testing variables at end of exercise between IRE and CWE.

\begin{tabular}{lrrr}
\hline \multicolumn{1}{c}{ Variables } & \multicolumn{1}{c}{ IRE } & \multicolumn{1}{c}{ CWE } & $P$ value \\
\hline$\dot{\mathrm{V}} \mathrm{O}_{2}(\mathrm{~L} / \mathrm{min})$ & $3.04 \pm 0.71$ & $3.10 \pm 0.77$ & n.s. \\
$\dot{\mathrm{VE}}(\mathrm{L} / \mathrm{min})$ & $99.1 \pm 25.2$ & $104.2 \pm 27.6$ & n.s. \\
$\mathrm{RER}$ & $1.22 \pm 0.11$ & $1.18 \pm 0.06$ & n.s. \\
$\mathrm{HR}(\mathrm{bpm})$ & $183.3 \pm 4.07$ & $183.7 \pm 7.31$ & n.s. \\
\hline
\end{tabular}

Variables are presented as mean \pm S.D. Each value was obtained at the end of exercise. IRE, incremental ramp exercise; CWE, constant work rate exercise; $\dot{\mathrm{V}} \mathrm{O}_{2}$, oxygen uptake; $\dot{\mathrm{VE}}$, minute ventilation; RER, respiratory exchange ratio; HR, heart rate; n.s., not significant.

the belly of the muscle approximately $10-14 \mathrm{~cm}$ above the knee joint, and the position was pen-marked to allow a similar re-positioning of the probe on subsequent appointments and to detect sliding of the probe during the tests. The probe was secured with elastic strapping after the skin had been carefully shaved. The instrument uses 3 lightemitting diodes (wavelengths of 780, 810, and $830 \mathrm{~nm}$ ) and calculates the tissue levels of $\mathrm{O}_{2} \mathrm{Hb}, \mathrm{HHb}$, and total hemoglobin ( $\mathrm{THb}$ ), according to the Beer-Lambert law. The data were monitored continuously, recorded second-by-second, and sampled at $10 \mathrm{~Hz}$ via a converter (NR500; Keyence, Osaka, Japan). The distances between the incident point and the 2 detectors were 30 and $15 \mathrm{~mm}$. We defined levels of oxygenation and deoxygenation in arbitrary units, and a physiological calibration of the $\mathrm{HHb}$ data was performed within 5 min after the exercise tests; data obtained during the exercise protocols were expressed as percentages of the maximal deoxygenation of the muscle, $100 \% \mathrm{HHb}$, as determined by pressure cuff inflation (at 300 $\mathrm{mmHg}$ ) at the root of the thigh until the HHb plateaued (Hamaoka et al. 1996) and the $0 \% \mathrm{HHb}$ was taken as a baseline value at rest in a seated position. We calculated muscle $\mathrm{O}_{2}$ saturation $\left(\mathrm{SmO}_{2}\right)$ from the
$\mathrm{O}_{2} \mathrm{Hb}$ and $\mathrm{THb}$ values with the following formula: $\mathrm{SmO}_{2}(\%)=$ $\left(\mathrm{O}_{2} \mathrm{Hb} / \mathrm{THb}\right) \times 100$. Adipose tissue thickness (ATT) has been reported to have a substantial confounding influence on in vivo NIRS measurements (van Beekvelt et al. 2001). We believe that ATT would influence the conversion of (780-830 nm) into oxygenation and deoxygenation concentration changes, using the Beer-Lambert law, but should not affect a kinetic study (Chuang et al. 2002).

\section{Statistical analyses}

Statistical analyses were performed using SPSS, version 19.0, statistical software (SPSS, Chicago, IL, USA). The peak value for each measurement was defined as the mean of the values acquired during the last $30 \mathrm{~s}$ of testing. For both tests, the HHb kinetics data, with respect to both the percentage of performance time and the percentage of the $\dot{\mathrm{V}}_{2 \max }$, were plotted to compare the tests. All data are presented as means \pm standard deviation (SD). Comparisons between the IRE and time-matched CWE results were analyzed using a paired $t$-test; $p<0.05$ was considered statistically significant. 

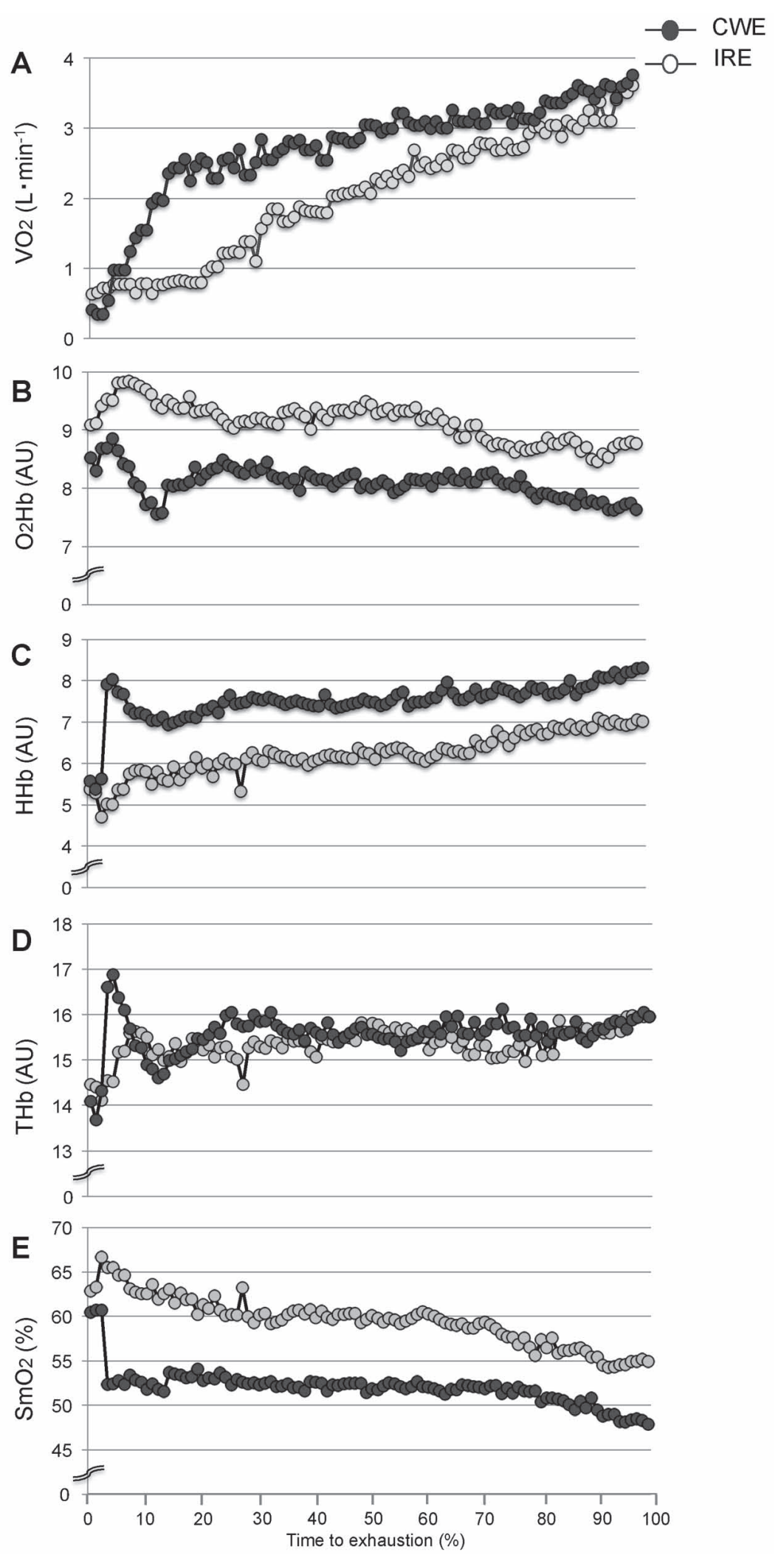

Fig. 2. Typical kinetics of physiological responses.

Typical kinetics of (A) oxygen uptake $\left(\dot{\mathrm{VO}}_{2}\right)$, (B) oxyhemoglobin $\left(\mathrm{O}_{2} \mathrm{Hb}\right),(\mathrm{C})$ deoxyhemoglobin $(\mathrm{HHb})$, (D) total hemoglobin (THb), and (E) muscle oxygen saturation $\left(\mathrm{SmO}_{2}\right)$ during incremental ramp exercise (IRE; $\circ$ ) and constant work-rate exercise (CWE; •), as percentages of the performance time; $0 \%$ values were averaged over the last $30 \mathrm{~s}$ of rest. AU, arbitrary units. 


\section{Results}

From the $\mathrm{P}-\mathrm{T}_{\lim }$ relationship, the WR of the timematched CWE was calculated to be $179.2 \pm 22.7 \mathrm{~W}(73.9 \%$ $\pm 5.5 \%$ of the $\left.\mathrm{WR}_{\text {peak }}\right)$. The performance time to exhaustion was not significantly different between CWE $(9.4 \pm 1.0$ $\mathrm{min})$ and IRE (9.6 $\pm 1.3 \mathrm{~min})$.

Table 2 shows a comparison of end-exercise ventilator responses during IRE and CWE. All participants completed the IRE by reaching exhaustion levels, with a $\mathrm{HR}_{\max }>90 \%$ $(95.1 \% \pm 3.11 \%)$ of the age-predicted value and an RER $>$ 1.15. There were no significant differences in $\dot{\mathrm{VO}}_{2}$. In addition, Fig. $2 \mathrm{~A}$ shows typical $\mathrm{V}_{2}$ kinetics as percentages of the performance time. During $\mathrm{CWE}, \dot{\mathrm{VO}}_{2}$ kinetics appeared to rise exponentially and to slowly reach (slow component) $\dot{\mathrm{V}} \mathrm{O}_{2 \max }$. During IRE, the $\dot{\mathrm{V}} \mathrm{O}_{2}$-Time relationship was approximately linear.

Fig. 2B-E show typical tracings for muscle oxygen- ation trends as percentages of the performance time; $\mathrm{O}_{2} \mathrm{Hb}$ (Fig. 2B) and $\mathrm{SmO}_{2}$ (Fig. 2E) during CWE rapidly decreased within the initial phase and continued to gradually decline until exhaustion, attaining values lower than in the IRE. The $\mathrm{THb}$ trends (Fig. 2C) were similar between the two tests. Fig. 3 shows the $\mathrm{HHb}$ kinetics as percentages of the performance time (Fig. 3A) and as percentages of $\dot{\mathrm{V}} \mathrm{O}_{2 \max }$ (Fig. 3B). The HHb levels at all time points, as percentages of the performance time, were significantly higher during CWE than during IRE $(p<0.05)$. On the other hand, $\mathrm{HHb}$ levels, as percentages of $\dot{\mathrm{V}} \mathrm{O}_{2 \max }$, were significantly higher during CWE than during IRE $(p<0.05)$, except at $80 \%$ of $\dot{\mathrm{V}} \mathrm{O}_{2 \max }$.

\section{Discussion}

This study compared muscle deoxygenation during IRE and CWE under similar conditions of exercise duration. The $\mathrm{HHb}$ levels during CWE exceeded those observed
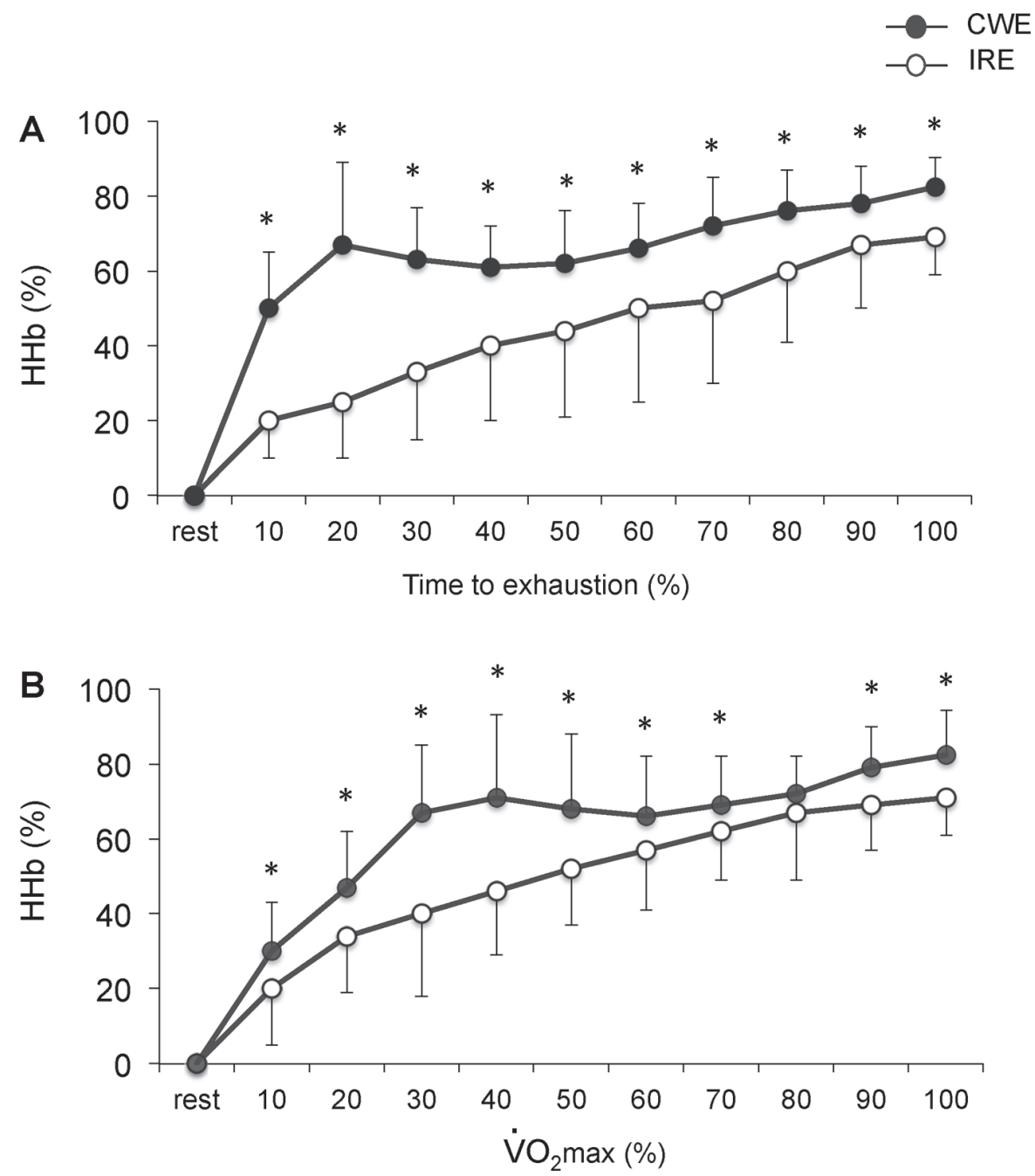

Fig. 3. Comparison of deoxyhemoglobin ( $\mathrm{HHb})$ kinetics between IRE and CWE.

The (A) deoxyhemoglobin ( $\mathrm{HHb}$ ) kinetics as percentages of the performance time and (B) HHb kinetics as percentages of maximum oxygen uptake $\left(\dot{\mathrm{VO}}_{2 \max }\right)$.

*Significantly different between incremental ramp exercise (IRE; ○) and constant work-rate exercise (CWE; •) at the same point $(p<0.05)$. 
during IRE, despite a non-significant difference between their $\dot{\mathrm{VO}}_{2}$ values. These results indicate that $\mathrm{CWE}$ extracts $\mathrm{O}_{2}$ at a rate comparable to that during IRE, and that the $\dot{\mathrm{V}}_{2 \text { max }}$ in CWE is linked to a greater overall $\mathrm{O}_{2}$ extraction. These data suggest that exercise capacity estimated using CWE shows a greater muscle $\mathrm{O}_{2}$ extraction capacity than when IRE is used.

Interestingly, the $\mathrm{HHb}$ kinetics, as percentages of the $\dot{\mathrm{V}} \mathrm{O}_{2 \max }$, showed that CWE produced higher values than IRE, except at $80 \%$ of $\dot{\mathrm{V}} \mathrm{O}_{2 \max }$. The higher $\mathrm{HHb}$ values during the initial phase of CWE, from $10 \%$ to $70 \%$ of $\dot{\mathrm{V}}_{2 \max }$, might result from excess $\mathrm{O}_{2}$ delivery, i.e., increased blood flow, compared with $\mathrm{O}_{2}$ utilization (Neary et al. 2001; DeLorey et al. 2003; Grassi et al. 2003) because of its higher WR during CWE. In the present study, differences in $\mathrm{HHb}$ values during the end phase, from $90 \%$ to $100 \%$ of $\dot{\mathrm{V}} \mathrm{O}_{2 \max }$, may be because of the "HHb slow component" during CWE (Jones et al. 2006; Bailey et al. 2009; DiMenna et al. 2010), a progressive increase in HHb throughout the steady state portion of the exercise, and "the plateau point" during IRE (Legrand et al. 2007; Boone et al. 2009; Osawa et al. 2011; Spencer et al. 2012). In the present study, the typical $\mathrm{HHb}$ kinetics (Fig. 2C) shows a progressive increase during CWE and the plateau during the end phase of IRE.

The discrepancy in muscle deoxygenation between CWE and IRE may be explained by the Bohr effect. Higher lactate concentrations are known to favor greater releases of oxygen from hemoglobin due to the rightward displacement of the $\mathrm{O}_{2} \mathrm{Hb}$ curve (Bohr effect), consequently facilitating $\mathrm{O}_{2}$ extraction from the blood (Stringer et al. 1994). In addition, the capacity to accumulate more lactate and produce more energy via anaerobic metabolism seems to be related to peripheral factors, such as the percentage of fast-twitch fibers. In agreement with this hypothesis, the HHb slow component has been reported to be strongly correlated with lactate concentrations during running (Ferri et al. 2012). Therefore, the CWE protocol elicits greater deoxygenation, probably because of greater lactic acidosis, and possibly changes the recruitment of different fiber types, favoring fast-twitch fibers.

The present study, however, could not elucidate the physiological mechanisms of the $\mathrm{HHb}$ slow component because of the study's limitations. These included a lack of data regarding blood lactate levels and the absence of electromyograms. Further research is necessary to clarify this discrepancy and to understand of the physiological mechanisms underlying the $\mathrm{HHb}$ slow component.

In conclusion, we compared muscle deoxygenation between IRE and CWE under similar conditions of exercise duration. In the present study, $\dot{\mathrm{V}} \mathrm{O}_{2}$ was not significantly different between the 2 exercise types, but $\mathrm{HHb}$ levels were higher during CWE than during IRE. These results suggest that skeletal muscles during CWE extract oxygen at a rate comparable to that during IRE under similar conditions in both tests, and that CWE-assessed exercise capacity might be linked to a higher overall $\mathrm{O}_{2}$ extraction. This discrep- ancy may be affected by the intramuscular metabolic conditions during exhaustion after both exercises, such as greater lactic acidosis and possible changes in fiber-type recruitment that favor fast-twitch fibers. The findings suggest that exercise tolerance estimated by CWE reflects a greater $\mathrm{O}_{2}$ extraction capacity, and that the fact that the CWE endurance time is more sensitive to rehabilitation intervention may be due to improvements in muscle oxygen extraction.

\section{Acknowledgements}

We are grateful to members of the Pulmonary Rehabilitation Laboratory of Kio University for their helpful suggestions.

\section{Conflict of Interest}

The authors declare no conflict of interest.

\section{References}

American Thoracic Society; American College of Chest Physicians (2003) ATS/ACCP statement on cardiopulmonary exercise testing. Am. J. Respir. Crit. Care Med., 167, 211-277.

Arizono, S., Taniguchi, H., Nishiyama, O., Kondoh, Y., Kimura, T., Kataoka, K., Ogawa, T., Watanabe, F., Nishimura, K., Senjyu, H. \& Tabira, K. (2011) Improvements in quadriceps force and work efficiency are related to improvements in endurance capacity following pulmonary rehabilitation in COPD patients. Intern. Med., 50, 2533-2539.

Bailey, S.J., Wilkerson, D.P., DiMenna, F.J. \& Jones, A.M. (2009) Influence of repeated sprint training on pulmonary $\mathrm{O}_{2}$ uptake and muscle deoxygenation kinetics in humans. J. Appl. Physiol., 106, 1875-1887.

Bassett, D.R. Jr. \& Howley, E.T. (2000) Limiting factors for maximum oxygen uptake and determinants of endurance performance. Med. Sci. Sports Exerc., 32, 70-84.

Boone, J., Koppo, K., Barstow, T.J. \& Bouckaert, J. (2009) Pattern of deoxy $[\mathrm{Hb}+\mathrm{Mb}]$ during ramp cycle exercise: influence of aerobic fitness status. Eur. J. Appl. Physiol., 105, 851-859.

Casaburi, R. (2005) Factors determining constant work rate exercise tolerance in COPD and their role in dictating the minimal clinically important difference in response to interventions. COPD, 2, 131-136.

Chuang, M.L., Ting, H., Otsuka, T., Sun, X.G., Chiu, F.Y., Hansen, J.E. \& Wasserman, K. (2002) Muscle deoxygenation as related to work rate. Med. Sci. Sports Exerc., 34, 1614-1623.

DeLorey, D.S., Kowalchuk, J.M. \& Paterson, D.H. (2003) Relationship between pulmonary $\mathrm{O}_{2}$ uptake kinetics and muscle deoxygenation during moderate-intensity exercise. J. Appl. Physiol., 95, 113-120.

DiMenna, F.J., Wilkerson, D.P., Burnley, M., Bailey, S.J. \& Jones, A.M. (2010) Priming exercise speeds pulmonary $\mathrm{O}_{2}$ uptake kinetics during supine "work-to-work" high-intensity cycle exercise. J. Appl. Physiol., 108, 283-292.

Ferri, A., Adamo, S., La Torre, A., Marzorati, M., Bishop, D.J. \& Miserocchi, G. (2012) Determinants of performance in 1,500-m runners. Eur. J. Appl. Physiol., 112, 3033-3043.

Grassi, B., Pogliaghi, S., Rampichini, S., Quaresima, V., Ferrari, M., Marconi, C. \& Cerretelli, P. (2003) Muscle oxygenation and pulmonary gas exchange kinetics during cycling exercise on-transitions in humans. J. Appl. Physiol., 95, 149-158.

Hamaoka, T., Iwane, H., Shimomitsu, T., Katsumura, T., Murase, N., Nishio, S., Osada, T., Kurosawa, Y. \& Chance, B. (1996) Noninvasive measures of oxidative metabolism on working human muscles by near-infrared spectroscopy. J. Appl. Physiol., 81, 1410-1417.

Hill, D.W., Poole, D.C. \& Smith, J.C. (2002). The relationship between power and the time to achieve $\dot{\mathrm{VO}}_{2 \max }$. Med. Sci. 
Sports Exerc., 34, 709-714.

Hogan, M.C., Richardson, R.S. \& Haseler, L.J. (1999) Human muscle performance and $\mathrm{PCr}$ hydrolysis with varied inspired oxygen fractions: a 31P-MRS study. J. Appl. Physiol., 86, $1367-1373$

Jones, A.M., Berger, N.J., Wilkerson, D.P. \& Roberts, C.L. (2006) Effects of "priming" exercise on pulmonary $\mathrm{O}_{2}$ uptake and muscle deoxygenation kinetics during heavy-intensity cycle exercise in the supine and upright positions. J. Appl. Physiol., 101, 1432-1441.

Jones, A.M., Wilkerson, D.P., DiMenna, F., Fulford, J. \& Poole, D.C. (2008) Muscle metabolic responses to exercise above and below the "critical power" assessed using 31P-MRS. Am. J. Physiol. Regul. Integr. Comp. Physiol., 294, R585-R593.

Legrand, R., Marles, A., Prieur, F., Lazzari, S., Blondel, N. \& Mucci, P. (2007) Related trends in locomotor and respiratory muscle oxygenation during exercise. Med. Sci. Sports Exerc., 39, 91-100.

Monod, H. \& Scherrer, J. (1965) The work capacity of a synergic muscular group. Ergonomics, 8, 329-338.

Moritani, T., Nagata, A., deVries, H.A. \& Muro, M. (1981) Critical power as a measure of physical work capacity and anaerobic threshold. Ergonomics, 24, 339-350.

Neary, J.P., Hall, K. \& Bhambhani, Y.N. (2001) Vastus medialis muscle oxygenation trends during a simulated $20-\mathrm{km}$ cycle time trial. Eur. J. Appl. Physiol., 85, 427-433.

Oga, T., Nishimura, K., Tsukino, M., Hajiro, T., Ikeda, A. \& Izumi, T. (2000) The effects of oxitropium bromide on exercise performance in patients with stable chronic obstructive pulmonary disease. A comparison of three different exercise tests. Am. J. Respir. Crit. Care Med., 161, 1897-1901.

Osawa, T., Kime, R., Hamaoka, T., Katsumura, T. \& Yamamoto, M. (2011) Attenuation of muscle deoxygenation precedes EMG threshold in normoxia and hypoxia. Med. Sci. Sports Exerc.,
43, 1406-1413.

Palange, P., Ward, S.A., Carlsen, K.H., Casaburi, R., Gallagher, C.G., Gosselink, R., O’Donnell, D.E., Puente-Maestu, L., Schols, A.M., Singh, S. \& Whipp, B.J. (2007) Recommendations on the use of exercise testing in clinical practice. Eur. Respir. J., 29, 185-209.

Poole, D.C., Ward, S.A., Gardner, G.W. \& Whipp, B.J. (1988) Metabolic and respiratory profile of the upper limit for prolonged exercise in man. Ergonomics, 31, 1265-1279.

Poole, D.C., Wilkerson, D.P. \& Jones, A.M. (2008) Validity of criteria for establishing maximal $\mathrm{O}_{2}$ uptake during ramp exercise tests. Eur. J. Appl. Physiol., 102, 403-410.

Sloniger, M.A., Cureton, K.J., Carrasco, D.I., Prior, B.M., Rowe, D.A. \& Thompson, R.W. (1996) Effect of the slow-component rise in oxygen uptake on $\mathrm{V}_{2 \max }$. Med. Sci. Sports Exerc., 28, $72-78$.

Spencer, M.D., Murias, J.M. \& Paterson, D.H. (2012) Characterizing the profile of muscle deoxygenation during ramp incremental exercise in young men. Eur. J. Appl. Physiol., 112, 3349-3360.

Stringer, W., Wasserman, K., Casaburi, R., Porszasz, J., Maehara, K. \& French, W. (1994) Lactic acidosis as a facilitator of oxyhemoglobin dissociation during exercise. J. Appl. Physiol., 76, 1462-1467.

van Beekvelt, M.C., Borghuis, M.S., van Engelen, B.G., Wevers, R.A. \& Colier, W.N. (2001) Adipose tissue thickness affects in vivo quantitative near-IR spectroscopy in human skeletal muscle. Clin. Sci., 101, 21-28.

Vanhatalo, A., Fulford, J., DiMenna, F.J. \& Jones, A.M. (2010) Influence of hyperoxia on muscle metabolic responses and the power-duration relationship during severe-intensity exercise in humans: a 31P magnetic resonance spectroscopy study. Exp. Physiol., 95, 528-540. 